\title{
Erratum: Hydrogeological research on intensively exploited deep aquifers in the 'Loma de Úbeda' area (Jaén, southern Spain)
}

Antonio González-Ramón • Javier Rodríguez-Arévalo •

Sergio Martos-Rosillo • Javier Gollonet

\section{Erratum to: Hydrogeology Journal (2013)}

DOI 10.1007/s10040-013-0957-4

For clarity, Table 1 and Table 3 are presented again here.

Table 1 Relationship between the stratigraphy and aquifers in the area

\begin{tabular}{|c|c|c|c|c|}
\hline SYSTEM & & $\begin{array}{l}\text { SERIES } \\
\text { AGE }\end{array}$ & LITHOLOGY & AQUIFER \\
\hline Quaternary & & $\begin{array}{l}\text { Holocene } \\
\text { Pleistocene }\end{array}$ & Alluvial & $\begin{array}{l}\text { Guadalquivir and Guadalimar } \\
\text { Alluvial }\end{array}$ \\
\hline \multirow{4}{*}{ Tertiary } & \multirow{3}{*}{ 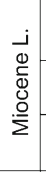 } & $\begin{array}{l}\text { Messinian- } \\
\text { Tortonian L. }\end{array}$ & $\begin{array}{l}\text { Conglomerate } \\
\text { and calcarenite }\end{array}$ & Upper Miocene aquifer \\
\hline & & Tortonian L. & $\begin{array}{l}\text { Marl, silt and } \\
\text { conglomerate }\end{array}$ & \\
\hline & & Tortonian E. & $\begin{array}{l}\text { Calcarenite and } \\
\text { calcareous breccia }\end{array}$ & Lower Miocene aquifer \\
\hline & & Miocene M. & Olistostromes & \\
\hline Jurassic & & Lias & $\begin{array}{l}\text { Dolomite and } \\
\text { Limestone }\end{array}$ & Jurassic aquifer \\
\hline \multirow{2}{*}{ Triassic } & & orian-Carnian & Clay and chalk marl & \\
\hline & & Ladinian & $\begin{array}{l}\text { Clay, sandstone and } \\
\text { conglomerate }\end{array}$ & Triassic aquifer \\
\hline \multirow{2}{*}{ Paleozoic } & & rboniferous L. & Granite & \\
\hline & & $\begin{array}{l}\text { arboniferous- } \\
\text { evonian }\end{array}$ & Shale and quartzite & \\
\hline
\end{tabular}

Published online: 6 April 2013

(C) Springer-Verlag Berlin Heidelberg 2013

The online version of the original article can be found at http://dx.doi.org/ 10.1007/s10040-013-0957-4.

A. González-Ramón (®) S. Martos-Rosillo

Geological Survey of Spain,

Urb Alcázar del Genil, 4 Edf. Zulema bajo, Granada, Spain

e-mail: antonio.gonzalez@igme.es

Tel.: +34-958-183143

Fax: +34-958-122990

S. Martos-Rosillo

e-mail: s.martos@igme.es

J. Rodríguez-Arévalo

Centre for Studies and Experimentation of Public Engineering,

CEDEX, C/Alfonso XII, 3, Madrid, Spain

e-mail: Javier.Rodriguez@cedex.es

J. Gollonet

C/. Torre de las Cabezas 1, esc. 3, $3^{\circ} \mathrm{D}$, Granada, Spain

e-mail: gollonet@terra.es 
Table 3 Main statistical parameters of water samples analyzed

\begin{tabular}{|c|c|c|c|c|c|c|c|c|}
\hline Aquifer (no. of samples) & Parameter & Unit & Maximum & Minimum & Average & Standard deviation & Variation Coefficient & Variance \\
\hline Miocene (93) & $\begin{array}{l}\mathrm{Ca} \\
\mathrm{Na} \\
\mathrm{Mg} \\
\mathrm{Cl} \\
\mathrm{SO}_{4} \\
\mathrm{HCO}_{3} \\
\mathrm{NO}_{3} \\
\mathrm{EC} \\
\text { Temp } \\
\mathrm{pH}\end{array}$ & $\begin{array}{l}\mathrm{mg} / 1 \\
\mathrm{mg} / 1 \\
\mathrm{mg} / 1 \\
\mathrm{mg} / 1 \\
\mathrm{mg} / 1 \\
\mathrm{mg} / 1 \\
\mathrm{mg} / 1 \\
\mu \mathrm{S} / \mathrm{cm} \\
{ }^{\circ} \mathrm{C}\end{array}$ & $\begin{array}{l}240 \\
329 \\
152 \\
133.6 \\
960 \\
607 \\
250 \\
2050 \\
27.6 \\
8.2\end{array}$ & $\begin{array}{c}20 \\
12 \\
15.2 \\
10 \\
27 \\
235 \\
0 \\
564 \\
13.5 \\
6.9\end{array}$ & $\begin{array}{c}90.5 \\
45.8 \\
53.7 \\
63.1 \\
144.3 \\
373.1 \\
69.4 \\
1060 \\
19.4 \\
7.5\end{array}$ & $\begin{array}{c}36.8 \\
42.6 \\
20.3 \\
29.2 \\
125.8 \\
77.7 \\
56.3 \\
245.5 \\
3 \\
0.312\end{array}$ & $\begin{array}{l}0.407 \\
0.97 \\
0.38 \\
0.46 \\
0.87 \\
0.208 \\
0.81 \\
0.232 \\
0.153 \\
0.042\end{array}$ & $\begin{array}{r}1355 \\
1813 \\
413 \\
851 \\
15821 \\
6032 \\
3165 \\
60269 \\
8.8 \\
0\end{array}$ \\
\hline Jurassic (198) & $\begin{array}{l}\mathrm{Ca} \\
\mathrm{Na} \\
\mathrm{Mg} \\
\mathrm{Cl} \\
\mathrm{SO}_{4} \\
\mathrm{HCO}_{3} \\
\mathrm{NO}_{3} \\
\mathrm{EC} \\
\mathrm{Temp} \\
\mathrm{pH}\end{array}$ & $\begin{array}{l}\mathrm{mg} / 1 \\
\mathrm{mg} / 1 \\
\mathrm{mg} / 1 \\
\mathrm{mg} / 1 \\
\mathrm{mg} / 1 \\
\mathrm{mg} / 1 \\
\mathrm{mg} / 1 \\
\mu \mathrm{S} / \mathrm{cm} \\
{ }^{\circ} \mathrm{C}\end{array}$ & $\begin{array}{c}208.3 \\
742 \\
100 \\
1360 \\
450 \\
597.8 \\
230 \\
5200 \\
55 \\
8.6\end{array}$ & $\begin{array}{c}9 \\
2 \\
5 \\
6 \\
10.5 \\
126 \\
0 \\
437 \\
15.3 \\
6.5\end{array}$ & $\begin{array}{r}75 \\
159.3 \\
39.8 \\
186.1 \\
131.6 \\
357.6 \\
30.8 \\
1412.3 \\
29.5 \\
7.4\end{array}$ & $\begin{array}{c}34.8 \\
181.8 \\
16.7 \\
250.9 \\
76.4 \\
77.6 \\
43.1 \\
799 \\
8.3 \\
0.393\end{array}$ & $\begin{array}{l}0.465 \\
1.1 \\
0.42 \\
1.3 \\
0.58 \\
0.217 \\
1.4 \\
0.566 \\
0.282 \\
0.05\end{array}$ & $\begin{array}{r}1214 \\
33045 \\
279 \\
62942 \\
5843 \\
6019 \\
1846 \\
638362 \\
69.5 \\
0\end{array}$ \\
\hline Triassic (29) & $\begin{array}{l}\mathrm{Ca} \\
\mathrm{Na} \\
\mathrm{Mg} \\
\mathrm{Cl} \\
\mathrm{SO}_{4} \\
\mathrm{HCO}_{3} \\
\mathrm{NO}_{3} \\
\mathrm{EC} \\
\text { Temp } \\
\text { pH }\end{array}$ & $\begin{array}{l}\mathrm{mg} / 1 \\
\mathrm{mg} / 1 \\
\mathrm{mg} / 1 \\
\mathrm{mg} / 1 \\
\mathrm{mg} / 1 \\
\mathrm{mg} / 1 \\
\mathrm{mg} / 1 \\
\mu \mathrm{S} / \mathrm{cm} \\
{ }^{\circ} \mathrm{C}\end{array}$ & $\begin{array}{c}440 \\
187.2 \\
202 \\
138.9 \\
1590 \\
479 \\
149.2 \\
2790 \\
24.6 \\
8.1\end{array}$ & $\begin{array}{c}58 \\
7 \\
41.5 \\
8.6 \\
198.6 \\
245 \\
7.6 \\
732 \\
15.7 \\
7.1\end{array}$ & $\begin{array}{r}181.9 \\
34 \\
106.3 \\
45.3 \\
587.4 \\
356.2 \\
33.1 \\
1550.1 \\
20.1 \\
7.6\end{array}$ & $\begin{array}{c}101.9 \\
45.7 \\
39.8 \\
44.2 \\
369.9 \\
61.8 \\
38.6 \\
506.9 \\
3 \\
0.335\end{array}$ & $\begin{array}{l}0.56 \\
1.4 \\
0.374 \\
0.98 \\
0.63 \\
0.173 \\
1.2 \\
0.327 \\
0.147 \\
0.04\end{array}$ & $\begin{array}{r}10384 \\
2089 \\
1581 \\
1956 \\
136792 \\
3815 \\
1486 \\
256932 \\
8.7 \\
0\end{array}$ \\
\hline Guadalimar River (70) & $\begin{array}{l}\mathrm{Ca} \\
\mathrm{Na} \\
\mathrm{Mg} \\
\mathrm{Cl} \\
\mathrm{SO}_{4} \\
\mathrm{HCO}_{3} \\
\mathrm{NO}_{3} \\
\mathrm{EC} \\
\text { Temp } \\
\text { pH }\end{array}$ & $\begin{array}{l}\mathrm{mg} / 1 \\
\mathrm{mg} / 1 \\
\mathrm{mg} / 1 \\
\mathrm{mg} / 1 \\
\mathrm{mg} / 1 \\
\mathrm{mg} / 1 \\
\mathrm{mg} / 1 \\
\mu \mathrm{S} / \mathrm{cm} \\
{ }^{\circ} \mathrm{C}\end{array}$ & $\begin{array}{c}103 \\
31 \\
75 \\
50 \\
226 \\
411.8 \\
104 \\
1203 \\
28.4 \\
8.5\end{array}$ & $\begin{array}{r}37 \\
7 \\
15 \\
14 \\
28 \\
170 \\
1 \\
420 \\
6.2 \\
7\end{array}$ & $\begin{array}{r}80.3 \\
17.8 \\
44.4 \\
27.9 \\
137.3 \\
287.6 \\
13.7 \\
812.4 \\
15.1 \\
8\end{array}$ & $\begin{array}{c}15 \\
5.2 \\
9.7 \\
8.4 \\
29.7 \\
59.5 \\
12.2 \\
167.4 \\
4.9 \\
0.32\end{array}$ & $\begin{array}{l}0.187 \\
0.292 \\
0.219 \\
0.3 \\
0.216 \\
0.207 \\
0.89 \\
0.206 \\
0.327 \\
0.04\end{array}$ & $\begin{array}{r}226 \\
27 \\
94 \\
70 \\
880 \\
3538 \\
148 \\
28024 \\
24.4 \\
0\end{array}$ \\
\hline Miocene-Jurassic (105) & $\begin{array}{l}\mathrm{Ca} \\
\mathrm{Na} \\
\mathrm{Mg} \\
\mathrm{Cl} \\
\mathrm{SO}_{4} \\
\mathrm{HCO}_{3} \\
\mathrm{NO}_{3} \\
\mathrm{EC} \\
\mathrm{Temp} \\
\mathrm{pH}\end{array}$ & $\begin{array}{l}\mathrm{mg} / 1 \\
\mathrm{mg} / 1 \\
\mathrm{mg} / 1 \\
\mathrm{mg} / 1 \\
\mathrm{mg} / 1 \\
\mathrm{mg} / 1 \\
\mathrm{mg} / 1 \\
\mu \mathrm{S} / \mathrm{cm} \\
{ }^{\circ} \mathrm{C}\end{array}$ & $\begin{array}{c}112.2 \\
2914 \\
152 \\
4400 \\
454 \\
666.1 \\
118 \\
15600 \\
44.5 \\
8.4\end{array}$ & $\begin{array}{c}19 \\
40 \\
11 \\
36 \\
23 \\
251 \\
0 \\
633 \\
21.7 \\
6.8\end{array}$ & $\begin{array}{r}46.6 \\
294.2 \\
33.4 \\
309.2 \\
166.5 \\
393.6 \\
12 \\
1795.2 \\
35.3 \\
7.4\end{array}$ & $\begin{array}{c}21.7 \\
390.1 \\
22.6 \\
610.6 \\
67.1 \\
70.5 \\
16.6 \\
1834.4 \\
6.9 \\
0.295\end{array}$ & $\begin{array}{l}0.465 \\
1.3 \\
0.676 \\
2 \\
0.403 \\
0.179 \\
1.4 \\
1 \\
0.195 \\
0.04\end{array}$ & $\begin{array}{r}469 \\
152143 \\
511 \\
372838 \\
4504 \\
4971 \\
277 \\
3364898 \\
47.1 \\
0\end{array}$ \\
\hline Jurassic-Triassic (71) & $\begin{array}{l}\mathrm{Ca} \\
\mathrm{Na} \\
\mathrm{Mg} \\
\mathrm{Cl} \\
\mathrm{SO}_{4} \\
\mathrm{HCO}_{3} \\
\mathrm{NO}_{3} \\
\mathrm{EC} \\
\mathrm{Temp} \\
\mathrm{pH}\end{array}$ & $\begin{array}{l}\mathrm{mg} / 1 \\
\mathrm{mg} / 1 \\
\mathrm{mg} / 1 \\
\mathrm{mg} / 1 \\
\mathrm{mg} / 1 \\
\mathrm{mg} / 1 \\
\mathrm{mg} / 1 \\
\mu \mathrm{S} / \mathrm{cm} \\
{ }^{\circ} \mathrm{C}\end{array}$ & $\begin{array}{c}564.3 \\
306.3 \\
152 \\
243.2 \\
1592.3 \\
399.6 \\
210 \\
3410 \\
30 \\
8\end{array}$ & $\begin{array}{c}47 \\
7.9 \\
41 \\
9.3 \\
142 \\
172 \\
0 \\
256 \\
18.8 \\
6.6\end{array}$ & $\begin{array}{r}169.3 \\
45.2 \\
69.5 \\
34.2 \\
492.5 \\
322.5 \\
26.3 \\
1343.3 \\
25 \\
7.3\end{array}$ & $\begin{array}{c}98.4 \\
52.8 \\
27 \\
37 \\
370.2 \\
57.9 \\
36.3 \\
585 \\
2.2 \\
0.3\end{array}$ & $\begin{array}{l}0.58 \\
1.2 \\
0.39 \\
1.1 \\
0.75 \\
0.18 \\
1.4 \\
0.436 \\
0.09 \\
0.04\end{array}$ & $\begin{array}{r}9680 \\
2789 \\
730 \\
1370 \\
137053 \\
3352 \\
1315 \\
342280 \\
5.0 \\
0\end{array}$ \\
\hline
\end{tabular}

